ESAIM: PROCEEDINGS, December 2009, Vol. 29, p. 1-7

F. Coquel, Y. Maday, S. Müller, M. Postel and Q. H. Tran, Editors

\title{
NEW TRENDS IN MULTIRESOLUTION AND ADAPTIVE METHODS FOR CONVECTION-DOMINATED PROBLEMS
}

\author{
Frédéric Coquel ${ }^{1,2}$, Yvon Maday ${ }^{1,2}$, Siegfried Müller ${ }^{3}$, Marie Postel ${ }^{1,2}$ and \\ QUANG HuY TRAN ${ }^{4}$
}

\begin{abstract}
The MAMCDP'09 workshop took place in Paris in January 2009. It was intended to promote multiresolution and other adaptive techniques for complex applications where convection is the prevailing phenomenon. Presentations were given by both senior and young researchers from various institutions over two days. In this introduction we summarize the presentations whose slides are available on the website of the workshop as well as the present contributions.
\end{abstract}

\section{Principles of ADAPtive MUltiResolution Methods For HyPeRbolic Systems}

The archetype of convection-dominated problems is modeled by a hyperbolic system of partial differential equations. In most physically relevant situations these equations are nonlinear and express balance laws. It is well known that the solution of such systems can develop singularities in finite time, even when starting from infinitely smooth initial conditions. As a consequence, in all hyperbolic systems - and to some extent in all convection-dominated problems - the computational domain is most likely to contain some spatially restricted but moving areas where the solution exhibits a locally strong variation.

The theoretical issues, as well as the numerical analysis of schemes for equations having singular solutions are steadily flourishing areas of research. Another issue is the computational aspect: in the neighbourhood of a singularity it is crucial to look for a fine resolution, in particular if the discontinuous nature of the solution prevents high-order schemes to be used. On the other hand, the fact that convection is dominating implies that this region moves in time. The size of the spatial domain involved in the computation may be too large to use a fine grid everywhere. Designing an adaptive mesh is, in this respect, a fundamental necessity. Furthermore, the adaptive mesh must be able to evolve in time along with the solution.

The first attempts to achieve this goal date back to the eighties, with the development of AMR methods on the principles introduced by Berger et al. [5,6]. At roughly the same time Harten $[47,48]$ started to explore multi-scale techniques based on wavelet theory as a tool for numerical analysis of hyperbolic PDEs. These approaches amount to taking advantage of the multi-scale decomposition of the solution in order to concentrate the representation of discontinuities (e.g., shocks) on a very fine grid in their vicinity. It is therefore possible to save computational resources (CPU time, memory) while preserving the accuracy of the initial scheme - or at least the same order of error.

\footnotetext{
1 UPMC Paris 06, UMR 7598 LJLL, Paris, F-75005 France

2 CNRS, UMR 7598 LJLL, Paris, F-75005 France

3 Institut für Geometrie und Praktische Mathematik, RWTH Aachen, Templergraben 55, 52056 Aachen, Germany

4 IFP, Département Mathématiques Appliquées, 1 et 4 avenue de Bois-Préau, 92852 Rueil-Malmaison, France
}

(C) EDP Sciences, SMAI 2009 
Let us summarize the principle in its broad outlines: one starts from a numerical scheme to solve a system of equations

$$
\partial_{t} u+\operatorname{div} f(u)=0
$$

A priori one can choose a finite difference scheme, a finite volume scheme, etc. The solution at a time $t^{n}$ is represented by discretized values $u_{k}^{n}$ on a grid $S_{J}$. In order to simplify the notations, the set $S_{j}$ also denotes the set of cell indices at the $j$ level. This grid has a mesh size of order $2^{-J}$, where $J$ is chosen sufficiently large so that $u_{k}^{n}$ with $k \in S_{J}$ represents a good approximation of the exact solution $u\left(t^{n}, x_{k}\right)$. The discrete values $\left(u_{k}^{n}\right)_{k \in S_{J}}$ of the approximate solution are updated from one time step to the other by evaluating fluxes through the interfaces between the mesh cells. The basic idea is to make a multi-scale decomposition of the solution, similar to a wavelet transform but based on a hierarchy of nested grids, and to use the coefficients of the transform as smoothness indicators. These indicators allow the grid to be adapted according to the local regularity of the solution - that is what we call the adaptive multiresolution. The solution is discretized on a grid made up of cells belonging to more or less refined levels of discretization according to the local smoothness. This grid or rather, the level locally used in the hierarchy of grids, evolves in time, since it depends on the solution. An advantage compared to the AMR methods, which also use smoothness indicators, is that the multi-scale transformation can be reversed at any time and that the solution is in fact known potentially on the finest grid, even if it is calculated on a much coarser one. This asset is very interesting from the point of view of the analysis of the method, which can thus be compared with the scheme of reference on the finest grid.

This method of adaptive multiresolution is a rather recent development $[26,46,49]$. Initially the work of $[10,47,48]$ rather concerned the use of the wavelet coefficients as error indicators in the context of an evolution scheme on the finest uniform grid. Indeed, the wavelet coefficients indicate zones where it is possible to save computing time on the calculation of fluxes, which is all the more expensive as the solution is singular: in the areas where the details beyond a level of scale $j<J$ are small (i.e. beneath a given threshold) the solution is considered as smooth and the costly evaluation of fluxes can be replaced by their interpolation starting from the values on a coarser level of grid. This is normally cheaper than the exact flux evaluation, in the case of conservation laws.

This has given rise to a great variety of adaptive multiresolution methods whose scope of applications is nowadays much larger than the initial hyperbolic systems. The goal of the MAMCDP'09 workshop was to review the state of the art in this area of research, and to identify important new trends.

\section{State of the ART AND EMERGing TRENDS}

Since the end of the nineties several teams have worked in these two directions with always the common objective to accelerate total calculation while keeping the same accuracy level as the reference scheme on a uniform grid. Multiresolution methods turn out to be very efficient. In particular, the adaptation process is only controlled by the threshold parameter. The choice of this parameter seems to be very robust with respect to varying configurations and applications. Ideally, it depends on the discretization error of the reference finite volume scheme. This was confirmed by rigorous mathematical estimates for scalar model problems.

Bihari et al. [9,11], developed the techniques suggested by Harten and applied them to complex systems and numerical schemes on Cartesian grids. Cohen et al. [25] adapted this method to unstructured triangular grids, whereas Dahmen et al. [35] realized it for curvilinear grids. Donat et al. [18-23,39] also followed this path, and adopted an encoding of the solution by its point values instead of by its mean values.

In the fully adaptive scope also several directions have been explored. On the basis of a tree data structure, Schneider et al. $[38,62,63]$ developed multidimensional applications of the adaptive algorithm for various classes of problems dominated by the convection but also including parabolic or reaction-diffusion terms. Cohen et al. [27-29] developed the fully adaptive algorithm on unstructured triangular grids for the compressible Euler equations. More recently Cohen et al. [24] combine multiresolution and anisotropy effects to design adaptive procedure on unstructured grids. Müller et al. have designed the adaptive algorithm on curvilinear grids for multidimensional applications, Euler compressible equations [12,55], shallow water equations [52], and cavitation 
bubbles [56]. It is explained in [55] how computing time efficiency is improved by means of a data structure using hash tables. Campos Pinto [16,17], Sonnendrücker and his co-authors $[7,8,68]$ have designed an adaptive method for the Vlasov-Poisson system in the context of a semi-Lagrangian formulation.

Using multiresolution analysis to model turbulence has also been explored by Farge et al. [43, 60, 66]. The inherent problem of simulation turbulent flows comes from the number of degrees of freedom needed to resolve turbulent structures. This number is proportional to $R e^{9 / 4}$ and becomes dramatically large with increasing Reynolds number $R e$ such as the ones used in aerodynamics $R e \approx 10^{6}$. This makes direct numerical simulations (DNS) impossible in many applications. However, in general, the interest is not in the fully resolved turbulent flow field but rather in some macroscopic quantities such as the lift and drag coefficients. These quantities can be resolved at the macro-scale although they are influenced by the non resolved fluctuations. Typically, the influence of these micro-scale fluctuations is described by algebraic models, like the Reynoldsaveraged Navier-Stokes equations (RANS) or the large eddy simulations (LES). Alternatively, the coherent vortex simulation (CVS) developed by Farge and Schneider $[44,67]$ for incompressible flows has been designed to compute this problem with a reduced number of degrees of freedom. This methodology is based on the wavelet representation of the vorticity. The basic idea is to extract the coherent vortex structures from the noise which will then be modeled to compute the flux evolution.

Initially adaptive multiresolution finite volume schemes have been derived only for explicit time discretizations. Bramkamp et al. show in [12] that it is possible to proceed similarly in case of an implicit time discretization. These are of interest when dealing with stationary flow problems, weakly unstationary problems or models that exhibit some stiffness due to relaxation processes, e.g. chemical reactions, or dissipation, e.g. diffusion, viscosity and heat conduction, resulting in anisotropic flow structures such as boundary layers. For these types of problems an explicit time discretization would lead to very small time steps in order to meet the CFL condition. Although the derivation is straight-forward, several new questions arise:

(i) In each time step the implicit time discretization results in a nonlinear system of discrete evolution equations. Typically this system is solved by Newton-Krylov methods. For steady state problems, only one Newton step is performed, because the time plays only the role of a relaxation parameter and there is no need to be accurate in each time step. However, for unstationary problems several Newton steps are needed to maintain the accuracy in each time step. In recent work by Steiner et al. [69,70], it was possible to design a break condition for the Newton methods that relies on the threshold value of the multi-scale method. To improve the efficiency of the solution of the nonlinear system one might employ the multilevel structure of the underlying grid hierarchy in the multi-scale analysis similar to adaptive multigrid techniques such as Brandt's so-called multilevel adaptive technique (MLAT) [13,14], that is an adaptive generalization of the full approximation scheme (FAS). The efficiency of these methods crucially relies on the proper choice of problem-dependent transfer and relaxation operators. Early investigations in [57] and [58] for unsteady state and steady state flow problems, respectively, show that contrary to classical adaptive multigrid schemes we may employ the multiresolution analysis using biorthogonal wavelets to define the restriction and prolongation operators. Since the underlying problem is nonlinear, the FAS [13] is used for the coarse grid correction. Further investigations are needed to fully employ the high potential of the multiresolution analysis when applying it directly to the discrete evolution equations arising from the finite volume discretization rather than just using it as a data compression tool for the set of discrete cell data.

(ii) By the implicit time discretization, the data in all cells are coupled and, hence, an information could propagate throughout the entire computational domain in one time step. Since the prediction strategy relies on the fact that the information propagates at most by one cell, the prediction has to be adjusted. Typically, for convection-dominated problems such as compressible fluid flow at high Reynolds numbers the influence of a local perturbation decays rapidly in space and stays more or less local. In [9], Bihari develops a heuristic approach for viscous problems where the parameter $q$ in Harten's strategy has been coupled with the viscosity parameter. However, a rigorous mathematical justification of its reliability is still missing. In [34] Coquel et al. tackle implicit-explicit schemes coupled with adaptive multiresolution 
mesh refinement. Here again the prediction of the tree should take into account the instantaneous propagation of information throughout the whole mesh, due to the implicit part of the scheme. However, intrinsic smoothing out of the singularities along with gradedness of the hierarchy of grids ensures that the same type of prediction of the tree as in the fully explicit case can be used.

Recently a new breakthrough in the design of a scheme adaptive both in time and space, due to Müller and Stiriba [57] gave rise to recent developments by Müller et al. [56], Schneider et al. [38] and Coquel et al. [31,32]. Time-space adaptivity in the context of parabolic problems is tackled by Faille and co-authors [41,42] in an approach combining domain decomposition and finite volume methods which makes a nice link with the AMR techniques. Another new trend is the control of the time-step in order to ensure the stability of the scheme throughout the simulation. For systems of equations, no maximum principle or invariant region is available. However, in some cases an explicit CFL-type condition can be derived [30], which should be satisfied at all time steps. It can then be incorporated in the local time stepping method to control [33, 59] the intermediate local time-steps. Other possible strategies have been investigated. The one investigated by Ferm et al. [45] stems out time step strategies for ODEs. A Runge-Kutta-Felhlberg method is applied to the semi-discretized flow equations by which the local spatial and temporal errors are estimated, allowing to determine the local step-size in space and time. Later on, Domingues et al. [37] also embedded this idea in fully adaptive multiresolution finite volume schemes. An alternative space-time adaptivity based upon Kuznetsov-type a posteriori $L_{1}$-error-estimates for scalar conservation laws is proposed by Kröner and Ohlberger [51]. More recently, explicit and implicit finite volume solvers on adaptively refined meshes have been coupled with adjoint techniques to control the time stepsizes for the solution of weakly unstationary compressible inviscid flow problems like transonic flight. These can be considered perturbations of stationary flows. While time accuracy is still needed to study phenomena like aeroelastic interactions, large time steps may be possible when the perturbations have passed. Here the time step control is based on a space-time-splitting of the adjoint error representation [3, 4, 40]. In [69, 70], the multi-scale-based grid adaptation was combined with these adjoint techniques to solve efficiently unstationary problems. The advantage of this space adaptive method is that it also provides an efficient break condition for the Newton iteration in the implicit time integration.

Another prominent line of research is the parallelization of multiresolution methods, in view of the ever increasing presence of parallel computers in the computing facilities [15,54,68]. Actually, even though multiscale based grid adaptation leads to a significant reduction of the computational complexity (CPU time and memory) in comparison to computations on uniform grids, this is not sufficient to efficiently perform realistic 3D computations. In addition, we need parallelization techniques in order to further reduce the computational time to an affordable order of magnitude. On a distributed memory architecture, the performance of a parallelized code crucially depends on the load-balancing and the inter-processor communications. Since the underlying adaptive grids are unstructured due to the presence of hanging nodes, this task is hardly trivial. For this purpose graph partitioning methods are frequently employed using the METIS Software [50]. An alternative approach is based on space-filling curves [72]. Here the basic idea is to map level-dependent multi-indices identifying the cells in a dyadic grid hierarchy of nested grids to a onedimensional line. The interval is then split into parts each containing the same number of entries, thus achieving optimal load balance. In the context of multiresolution schemes, both the graph-partitioning [53,64,65] and the space-filling curves [2,15] approaches have been used. Here again the comparison with AMR techniques is valuable. In most AMR configurations the adaptive mesh is already subdivided into patches. This inherent domain decomposition is obviously to be taken into account when going to a parallel architecture. A major difference is that the adaptive grids are not varying at each time step as is the case in multiresolution.

The need to study the links between Adaptive Mesh Refinement and multiresolution analysis has in fact been a major key-point of this workshop. In the original AMR methods developed by Berger et al. in the 1980's, the refinement process is triggered by gradients [61] or higher-order interpolation [1]. Recent investigations by Donat et al. [39] show that using a discrete multiresolution analysis instead leads to a much more efficient refinement criterion. In particular, in areas of partial smoothness such as rarefaction waves. It turned out that only minimal changes in the existing AMR code were necessary to embed the multiresolution-based 
refinement criterion. A systematic comparison of the performances of the fully adaptive multiresolution method and the adaptive mesh refinement method was investigated by Deiterding et al. [36]. For a 2D compressible Euler test case, the results obtained by both methods with similar accuracy requirements are compared in terms of computational overhead and compression of the computational grid, using in addition either local or global time stepping strategies. The conclusion is that the multiresolution techniques yield improved memory compression and gain in CPU time with respect to the adaptive mesh refinement method. Extending far beyond the elliptic equations community, AMR methods in the Galerkin framework are also developed for unstationary problems like convection-reaction-diffusion [71] using a posteriori error estimates to monitor the mesh adaptation. Here again the comparison with multiresolution adaptive methods might lead to interesting developments.

To sum it up, the MAMCDP09 workshop along with its proceedings provide an overview of the important developments in multiresolution adaptative methods. New trends have been identified: local time stepping, time step control, implicit schemes, parallelism. The latter generates also a lot of ongoing activity in the AMR community. The need for strong interaction between Computer Science and Mathematic communities has been emphasized in many presentations. Close collaboration is certainly necessary in this direction of research where the choice of the data structures and the program structure are of the utmost importance.

\section{REFERENCES}

[1] A. Baeza and P. Mulet. Adaptive mesh refinement techniques for high-order shock capturing schemes for multi-dimensional hydrodynamic simulations. Int. J. Numer. Meth. Fluids, 52(4):455-471, 2006.

[2] J. Ballmann, M. Behr, K. Brix, W. Dahmen, C. Hohn, R. Massjung, S. S. Melian, S. Müller, and G. Schieffer. Parallel and adaptive methods for fluid-structure interactions. Report No. 296, October 2009, IGPM, RWTH Aachen, 2009.

[3] R. Becker and R. Rannacher. A feed-back approach to error control in finite element methods: basic analysis and examples. East-West J. Numer. Math., 4(4):237-264, 1996.

[4] R. Becker and R. Rannacher. An optimal control approach to a posteriori error estimation in finite element methods. Acta Numer., 10:1-102, 2001.

[5] M. Berger and J. Oliger. Adaptive mesh refinement for hyperbolic partial differential equations. J. Comput. Phys., 53:484-512, 1984.

[6] M. Berger and P. Collela. Local adaptive mesh refinement for shock hydrodynamics. J. Comput. Phys., 82:64-84, 1989.

[7] N. Besse, F. Filbet, M. Gutnic, I. Paun, and E. Sonnendrücker. An adaptive numerical method for the Vlasov equation based on a multiresolution analysis. In F. Brezzi, A. Buffa, S. Escorsaro, and A. Murli, editors, Numerical Mathematics and Advanced Applications ENUMATH 2001, pages 437-446. Springer, 2001.

[8] N. Besse, G. Latu, A. Ghizzo, E. Sonnendrücker, and P. Bertrand. A wavelet-MRA-based adaptive semi-Lagrangian method for the relativistic Vlasov-Maxwell system. J. Comput. Phys., 227(16):7889-7916, 2008.

[9] B. L. Bihari. Multiresolution schemes for conservation laws with viscosity. J. Comput. Phys., 123(1):207-225, 1996.

[10] B. L. Bihari and A. Harten. Application of generalized wavelets: an adaptive multiresolution scheme. J. Comput. Appl. Math., 61(3):275-321, 1995.

[11] B. L. Bihari and D. Schwendeman. Multiresolution schemes for the reactive Euler equations. J. Comput. Phys., 154(1):197-230, 1999.

[12] F. Bramkamp, P. Lamby, and S. Müller. An adaptive multiscale finite volume solver for unsteady and steady state flow computations. J. Comput. Phys., 197(2):460-490, 2004.

[13] A. Brandt. Multi-level adaptive solutions to boundary-value problems. Math. Comp., 31(138):333-390, 1977.

[14] A. Brandt. Multi-level adaptive techniques (MLAT) for partial differential equations: ideas and software. In Mathematical software, III (Proc. Sympos., Math. Res. Center, Univ. Wisconsin, Madison, Wis., 1977), pages 277-318. Publ. Math. Res. Center, No. 39. Academic Press, New York, 1977.

[15] K. Brix, S. Melian, S. Müller, and G. Schieffer. Parallelisation of multiscale-based grid adaptation using space-filling curves. In F. Coquel, Y. Maday, S. Müller, M. Postel, and Q. H. Tran, editors, Multiresolution and Adaptive Methods for ConvectionDominated Problems, volume 29 of ESAIM Proceedings, Paris, 2009. EDP Sciences, Les Ulis.

[16] M. Campos Pinto. How to predict accurate wavelet grids in adaptive semi-Lagrangian schemes? In F. Coquel, Y. Maday, S. Müller, M. Postel, and Q. H. Tran, editors, Multiresolution and Adaptive Methods for Convection-Dominated Problems, volume 29 of ESAIM Proceedings, Paris, 2009. EDP Sciences, Les Ulis.

[17] M. Campos Pinto and M. Mehrenberger. Convergence of an adaptive scheme for the one-dimensional Vlasov-Poisson system. Prepublication INRIA RR-5519, 2005.

[18] G. Chiavassa and R. Donat. Numerical experiments with point value multiresolution for 2D compressible flows. Technical report, GrAN-99-4, University of Valencia, 1999. 
[19] G. Chiavassa and R. Donat. Point value multiscale algorithms for 2D compressible flows. SIAM J. Sci. Comput., 23(3):805-823, 2001.

[20] G. Chiavassa and R. Donat. Numerical experiments with multilevel schemes for conservation laws. In E.F. Toro, editor, Godunov methods - Theory and Applications, pages 155-160. Kluwer/Plenum Press, 2002.

[21] G. Chiavassa, R. Donat, and A. Marquina. Fine-mesh numerical simulations for 2D Riemann problems with a multilevel scheme. In H. Freistühler and G. Warnecke, editors, Hyperbolic problems: theory, numerics, applications, Vol. I, II (Magdeburg, 2000), volume 140/141 of Internat. Ser. Numer. Math., pages 247-256. Birkhäuser, Basel, 2001.

[22] G. Chiavassa and J. Liandrat. A fully adaptive wavelet algorithm for parabolic partial differential equations. Appl. Numer. Math., 36(2-3):333-358, 2001.

[23] G. Chiavassa. Utilisation of Harten multiresolution in scientific computing: two examples. MAMCDP09 workshop, http://www. ann.jussieu.fr/mamcdp09/slides/GuillaumeChiavassaMAMCDP09.pdf.

[24] A. Cohen. Combining multiresolution and anisotropy: theory, algorithms and open problems. http://www.ann.jussieu.fr/ mamcdp09/slides/AlbertCohenMAMCDP09.pdf. MAMCDP09 workshop.

[25] A. Cohen, N. Dyn, S. M. Kaber, and M. Postel. Multiresolution schemes on triangles for scalar conservation laws. J. Comput. Phys., 161(1):264-286, 2000.

[26] A. Cohen, S. M. Kaber, S. Müller, and M. Postel. Fully adaptive multiresolution finite volume schemes for conservation laws. Math. Comp., 72(241):183-225, 2003.

[27] A. Cohen, S. M. Kaber, and M. Postel. Multiresolution analysis on triangles: application to conservation laws. In Finite Volumes for Complex Applications II, pages 841-848. Hermes Sciences, 1999.

[28] A. Cohen, S. M. Kaber, and M. Postel. Multiresolution analysis on triangles: application to gas dynamics. In H. Freistühler and G. Warnecke, editors, Hyperbolic problems: theory, numerics, applications, Vol. I, II (Magdeburg, 2000), volume 140/141 of Int. Ser. Numer. Math., pages 257-266. Birkhäuser, Basel, 2001.

[29] A. Cohen, S. M. Kaber, and M. Postel. Adaptive multiresolution for finite volume solutions of gas dynamics. Computer and Fluids, 32:31-38, 2003.

[30] F. Coquel, Q. L. Nguyen, M. Postel, and Q. H. Tran. Entropy-satisfying relaxation method with large time-steps for Euler IBVPs. Prepublication LJLL R08004, to appear, 2007.

[31] F. Coquel, Q. L. Nguyen, M. Postel, and Q. H. Tran. Local time stepping applied to implicit-explicit methods for hyperbolic systems. Prepublication LJLL R07058, to appear, 2007.

[32] F. Coquel, Q. L. Nguyen, M. Postel, and Q. H. Tran. Local time stepping for implicit-explicit methods on time varying grids. 2007. Proceedings of ENUMATH 2007.

[33] F. Coquel, Q. L. Nguyen, M. Postel, and Q. H. Tran. Local time stepping with adaptive time step control for a two-phase fluid system. In F. Coquel, Y. Maday, S. Müller, M. Postel, and Q. H. Tran, editors, Multiresolution and Adaptive Methods for Convection-Dominated Problems, volume 29 of ESAIM Proceedings, Paris, 2009. EDP Sciences, Les Ulis.

[34] F. Coquel, M. Postel, N. Poussineau, and Q. H. Tran. Multiresolution technique and explicit-implicit scheme for multicomponent flows. J. Numer. Math., 14(3):187-216, 2006. In A. Kunoth and S. Müller, Special Issue on Breaking Complexity: Multiscale Methods for Efficient PDE Solvers. See also R05026, prepublication LJLL, Paris.

[35] W. Dahmen, B. Gottschlich-Müller, and S. Müller. Multiresolution schemes for conservation laws. Numer. Math., 88(3):399443, 2001.

[36] R. Deiterding, M.-O. Domingues, S. Gomes, O. Roussel, and K. Schneider. Adaptive multiresolution or adaptive mesh refinement? A case study for 2D Euler equations. In F. Coquel, Y. Maday, S. Müller, M. Postel, and Q. H. Tran, editors, Multiresolution and Adaptive Methods for Convection-Dominated Problems, volume 29 of ESAIM Proceedings, Paris, 2009. EDP Sciences, Les Ulis.

[37] M. Domingues, O. Roussel, and K. Schneider. On space-time adaptive schemes for the numerical solution of PDEs. In CEMRACS 2005-computational aeroacoustics and computational fluid dynamics in turbulent flows, volume 16 of ESAIM Proc., pages 181-194. EDP Sciences, Les Ulis, 2007.

[38] M. O. Domingues, S. M. Gomes, O. Roussel, and K. Schneider. An adaptive multiresolution scheme with local time stepping for evolutionary PDEs. J. Comput. Phys., 227(8):3758-3780, 2008.

[39] R. Donat. Using Harten's multiresolution framework on existing codes for hyperbolic PDEs. http://www.ann.jussieu.fr/ mamcdp09/slides/RosaDonatMAMCDP09.pdf. MAMCDP09 workshop.

[40] K. Eriksson and C. Johnson. Adaptive finite element methods for parabolic problems. IV. Nonlinear problems. SIAM J. Numer. Anal., 32(6):1729-1749, 1995.

[41] I. Faille. Local time steps for a finite volume scheme. http://www.ann.jussieu.fr/mamcdp09/slides/IsabelleFailleMAMCDP09.pdf. MAMCDP09 workshop.

[42] I. Faille, F. Nataf, F. Willien, and S. Wolf. Two local time stepping schemes for parabolic problems. In F. Coquel, Y. Maday, S. Müller, M. Postel, and Q. H. Tran, editors, Multiresolution and Adaptive Methods for Convection-Dominated Problems, volume 29 of ESAIM Proceedings, Paris, 2009. EDP Sciences, Les Ulis.

[43] M. Farge. Wavelet-based CVS method to solve a convection-dominated problem: the numerical simulation of turbulence. http://www.ann.jussieu.fr/mamcdp09/slides/MarieFargeMAMCDP09.pdf. MAMCDP09 workshop. 
[44] M. Farge and K. Schneider. Coherent vortex simulation (CVS), a semi-deterministic turbulence model using wavelets. Flow Turbul. Combust., 66(4):393-426, 2001.

[45] L. Ferm and P. Lötstedt. Space-time adaptive solution of first order PDEs. J. Sci. Comput., 26(1):83-110, 2006.

[46] B. Gottschlich-Müller and S. Müller. Adaptive finite volume schemes for conservation laws based on local multiresolution techniques. In M. Fey et al., editors, Hyperbolic problems: Theory, numerics, applications, Vol. I (Zürich, 1998), volume 129 of Int. Ser. Numer. Math., pages 385-394. Birkhäuser, Basel, 1999.

[47] A. Harten. Adaptive multiresolution schemes for shock computations. J. Comput. Phys., 115:319-338, 1994.

[48] A. Harten. Multiresolution algorithms for the numerical solutions of hyperbolic conservation laws. Comm. on Pure and Appl. Math., 48:1305-1342, 1995.

[49] M. K. Kaibara and S. M. Gomes. A fully adaptive multiresolution scheme for shock computations. Toro, E. F. (ed.), Godunov methods. Theory and applications. International conference, Oxford, GB, October 1999. New York, NY: Kluwer Academic/ Plenum Publishers. 497-503 (2001)., 2001.

[50] G. Karypis and V. Kumar. A fast and high quality multilevel scheme for partitioning irregular graphs. SIAM J. Sci. Comput., 20(1):359-392 (electronic), 1998.

[51] D. Kröner and M. Ohlberger. A posteriori error estimates for upwind finite volume schemes for nonlinear conservation laws in multidimensions. Math. Comp., 69(229):25-39, 2000.

[52] P. Lamby, S. Müller, and Y. Stiriba. Solution of shallow water equations using fully adaptive multiscale schemes. Int. J. Numer. Meth. Fluids, 49(4):417-437, 2005.

[53] M. Mehrenberger, E. Violard, O. Hoenen, M. Campos Pinto, and M. Sonnendrücker. A parallel adaptive Vlasov solver based on hierarchical finite element interpolation. Nuclear Instruments and Methods in Physics Research A, 558(1):188-191, 2006.

[54] S. Mogosan. Parallelization of multiscale-based grid adaptation using space filling curves. http://www.ann.jussieu.fr/mamcdp09/ slides/SoranaMogosanMAMCDP09.pdf. MAMCDP09 workshop.

[55] S. Müller. Adaptive Multiscale Schemes for Conservation Laws, volume 27 of Lecture Notes on Computational Science and Engineering. Springer, 2002.

[56] S. Müller, P. Helluy, and J. Ballmann. Numerical simulation of cavitation bubbles by compressible two-phase fluids. Int. J. Numer. Meth. Fluids, DOI:10.1002/fld.2033, 2009. Report No. 273, IGPM, RWTH Aachen, 2007.

[57] S. Müller and Y. Stiriba. Fully adaptive multiscale schemes for conservation laws employing locally varying time stepping. $J$. Sci. Comput., 30, No. 3:493-531, 2007. Report No. 238, IGPM, RWTH Aachen.

[58] S. Müller and Y. Stiriba. A multilevel finite volume method with multiscale-based grid adaptation for steady compressible flows. J. Comput. Appl. Math., 227(2):223-233, 2009.

[59] Q. L. Nguyen. A new strategy for adapting time-step in the local time stepping method applied to hyperbolic PDEs. http: //www.ann.jussieu.fr/mamcdp09/slides/QuangLongNguyenMAMCDP09.pdf. MAMCDP09 workshop.

[60] R. Nguyen van yen and M. Farge and K. Schneider. Wavelet regularization of a Fourier-Galerkin method for solving the 2d incompressible Euler equations. In F. Coquel, Y. Maday, S. Müller, M. Postel, and Q. H. Tran, editors, Multiresolution and Adaptive Methods for Convection-Dominated Problems, volume 29 of ESAIM Proceedings, Paris, 2009. EDP Sciences, Les Ulis.

[61] J. J. Quirk. A parallel adaptive grid algorithm for computational shock hydrodynamics. Appl. Numer. Math., 20(4):427-453, 1996. Adaptive mesh refinement methods for CFD applications (Atlanta, GA, 1994).

[62] O. Roussel, K. Schneider, and M. Farge. Coherent vortex extraction in 3D homogeneous turbulence: comparison between orthogonal and biorthogonal wavelet decompositions. J. Turbul., 6(11):1-15, 2005.

[63] O. Roussel, K. Schneider, A. Tsigulin, and H. Bockhorn. A conservative fully adaptive multiresolution algorithm for parabolic PDEs. J. Comput. Phys., 188:493-523, 2003.

[64] O. Roussel and K. Schneider. An adaptive multiresolution method for combustion problems: application to flame ball. Computers and Fluids, 34(7):817-831, 2005.

[65] O. Roussel and K. Schneider. Numerical study of thermodiffusive flame structures interacting with adiabatic walls using an adaptive multiresolution scheme. Combust. Theory Model., 10(2):273-288, 2006.

[66] K. Schneider. Fully adaptive multiresolution methods for evolutionary PDEs. http://www.ann.jussieu.fr/mamcdp09/slides/ KaiSchneiderMAMCDP09.pdf. MAMCDP09 workshop.

[67] K. Schneider and M. Farge. Adaptive wavelet simulation of a flow around an impulsively started cylinder using penalisation. Appl. Comput. Harmon. Anal., 12(3):374-380, 2002.

[68] E. Sonnedrücker. Adaptive methods for the Vlasov equation. http://www.ann.jussieu.fr/mamcdp09/slides/ EricSonnendruckerMAMCDP09.pdf. MAMCDP09 workshop.

[69] Ch. Steiner. Adaptive timestepping for conservation laws via adjoint error representation. PhD thesis, RWTH Aachen, 2008. http://darwin.bth.rwth-aachen.de/opus3/volltexte/2009/2679/.

[70] Ch. Steiner, S. Müller, and S. Noelle. Adaptive timestep control for weakly instationary solutions of the Euler equations. IGPM-Report 292, RWTH Aachen, 2009.

[71] M. Vohralìk. Guaranteed and robust discontinuous galerkin a posteriori error estimates for convection-diffusion-reaction problems. http://www.ann.jussieu.fr/mamcdp09/slides/MartinVohralikMAMCDP09.pdf. MAMCDP09 workshop.

[72] G. Zumbusch. Parallel Multilevel Methods. Adaptive Mesh Refinement and Loadbalancing. Teubner, 2003. 